\title{
Normal Wiggly Probabilistic Hesitant Fuzzy Information for Environmental Quality Evaluation
}

\author{
Qasim Noor (D), Dalal Awadh Alrowaili (iD, ${ }^{2}$ Tabasam Rashid (D), \\ and Syed Muhammad Husnine ${ }^{1}$ \\ ${ }^{1}$ Department of Science and Humanities, National University of Computer and Emerging Sciences, Lahore, Pakistan \\ ${ }^{2}$ Mathematics Department, College of Science, Jouf University, P.O. Box: 2014, Sakaka, Saudi Arabia \\ ${ }^{3}$ University of Management and Technology, Lahore, Pakistan \\ Correspondence should be addressed to Tabasam Rashid; tabasam.rashid@gmail.com
}

Received 5 June 2021; Revised 8 October 2021; Accepted 19 October 2021; Published 17 November 2021

Academic Editor: Atila Bueno

Copyright (c) 2021 Qasim Noor et al. This is an open access article distributed under the Creative Commons Attribution License, which permits unrestricted use, distribution, and reproduction in any medium, provided the original work is properly cited.

As a valuable tool for representing uncertain information, probabilistic hesitant fuzzy sets (PHFS) have gained considerable recognition and in-depth discussion in recent years to increase the flexibility and manifest hesitant information in decisionmaking problems. However, decision-makers (DMs) cannot express all preferences only through a few probabilistic terms in actual decision-making. Much critical information is hidden behind the original information provided by the DMs. Keeping that in mind, we are interested in mining deeper uncertain information from the original probabilistic hesitant fuzzy evaluation data. To achieve the target, we put forward a novel representation tool called the normal wiggly probabilistic hesitant fuzzy set (NWPHFS) to extract deeper uncertain preferences from original probabilistic information. NWPHFS retains the original evaluation information and carries and assesses the potential uncertain details for increasing the rationality of decision-making outcomes. Herein, we propose some fundamental concepts of NWPHFS, for instance, some elementary operational laws, distance measures between two NWPHFSs, and score function. We also suggest two new aggregation operators, that is, the normal wiggly probabilistic hesitant fuzzy weighted averaging (NWPHFWA) and normal wiggly probabilistic hesitant fuzzy weighted geometric (NWPHFWG). A novel mechanism is proposed here to work out multiattribute decision-making (MADM) in solving normal wiggly probabilistic decision-making problems. Through a practical example of environmental quality assessment, the specific calculation steps of this method are epitomized. Finally, we have demonstrated the feasibility and advancement of the proposed approach via a comprehensive comparative study.

\section{Introduction}

MADM problems are everywhere in our daily lives, and most people frequently face uncertain decision-making in all aspects of their lives, for example, which city to travel during a short holiday, which bag is suitable for shopping today, which mobile phone brand is more suitable for my needs, which kind of fruits to buy, and which clothes to wear today. While these common decision-making issues are easy to handle, no matter how many final choices are made, no errors can be significantly highlighted in MADM applications. However, no mistake can be tolerated in significant decision-making issues. The best or optimal decisions must be made for applications, including market analysis, quality assessment, and investment strategy. To address these problems, many effective representational models have been proposed and widely implemented, especially for MADM $[1,2]$. Almost all decisions take several steps to reach the final destination, and some of them can be confusing in nature. Suppose that the data is analyzed without handling the uncertainty. In that case, their decision outcomes may be extremely vague, so it is important to include the DM's preference to deal with uncertainty. Fuzzy set (FS) theory [3] and its extensions, such as intuitionistic fuzzy set (IFS) [4], type-2 fuzzy sets [5], interval-valued intuitionistic fuzzy set [6], Pythagorean fuzzy set [7], hesitant fuzzy set (HFS) [8], 
probabilistic HFS (PHFS) [9], and proportional HFS (PrHFS) [10], are the most effective tools to deal with the impreciseness and uncertainties in the decision-making. The purpose of the above-mentioned different types of fuzzy sets is to more effectively express information on the uncertain and complex diagnosis of DM. In recent decades, these generalizations of fuzzy sets have been widely recognized in various academic studies, and considerable achievements have been made to adapt to different application environments. In FS, DMs only evaluate the objects by stating the degree of membership (preferences), or they can only provide their assessment in a crisp value. However, for DMs, the key issue in dealing with a real complex problem is determining the crisp value based on a given standard to reflect their given uncertainty and opacity. For example, suppose that the DM cannot determine which specific number to give for object/alternative under a particular attribute. In that case, he may give several numbers instead of the specific number to represent his assessment information. Keeping this fact in mind, among these extensions, the most famous generalization of the FS is HFS [11], in which membership of a particular element is allowed to represent a set possessing many possible values between $[0,1]$. Therefore, HFS has a wider range of applications and more practical significance and is a handy tool for expressing people's hesitation in everyday life compared to other extensions. As it leads to expressing uncertainty, it has attracted the attention of researchers. For instance, Zhang [12] proposed different aggregation operators and several useful properties and discussed the relationship between them. Li et al. [13] developed a variety of novel similarities and distance measures between HFSs, in which both the values and the number of values of HFE are taken into account. Liu et al. [14] elucidated the correlation and distance measures for hesitant fuzzy information and analyzed their properties to measure the strength of the relationship between HFSs.

Since its initiation, several researchers have proposed a lot of research to support HFS theory [15-17]. In numerous decision-making problems, information is mainly vague or ambiguous because of the inaccurate/incomplete data, lack of time, partial attention, and the information processing skills of the decision-makers. Therefore, it is difficult for DMs to express their opinions in some specific numerical values. For instance, suppose that a consumer wants to buy a car. He mainly focuses on car safety features and asks an expert for advice. If the total is 100 points, the expert is $80 \%$ sure that the car's safety could be 60 , and he is $20 \%$ sure that the score could be 70 . The HFS $\{0.6,0.7\}$ cannot represents the preferences that 0.7 is more suitable than 0.6. To cope with the situation, Zhu [9] merged the probability into the HFS and proposed PHFS, which can cover the expert's hesitations and retain more information than HFS. For example, PHFS for the above case can be written as $\{0.6(0.8), 0.7(0.2)\}$, where 0.8 and 0.2 are the probabilities to the original HFE values, which can be employed to characterize DMs preferences. Zhang et al. [18] proposed the novel aggregation operators and continuous form for the improved PHFS and PHFE. Li et al. [19] proposed the
MADM method with PHF information based on the dominance degree of probabilistic hesitant fuzzy elements (PHFE) and the best-worst method. Wang and Li [20] studied PHFS operations to explore MADM problems and introduced an approach based on correlation coefficients that utilize probabilistic hesitant fuzzy information.

The development of various extension forms of PHFS supports DMs to articulate their assessment information about alternatives comprehensively. For instance, the interval-valued probabilistic hesitant fuzzy set (IVPHFS) presented by Krishankumar et al. [21] permits DMs to allot the probable values in interval forms. Also, Krishankumar et al. [22] discussed the IVPHFS under context when the weights of the attributes and DMs are unknown. The weights of the attributes are calculated using the interval-valued probabilistic hesitant deviation method. In contrast, the Bayesian approximation method is used to find the weights of the DMs under the environment of IVPHFS. IVPHFS is more likely to ask DMs to provide cognitive information through probabilistic hesitant fuzzy information and then ask them to further improve uncertainty assessments in various extension forms. Recently Noor et al. [23] proposed a new MADM method (tail decision-making) to find the best alternative by using the minimal information of the probabilistic interval-valued HFS. Chen et al. [24] proposed ordered weighted averaging operators generation algorithm for MADM problems. Xiong et al. [25] presented an extended power average operators for decision-making problems. However, these extensions become more complicated, which will increase the time cost and psychological burden of DM. It becomes difficult for us to evaluate the DMs' values, which they want to elaborate. Thus, obtaining more thorough investigation information to ensure the validity of the final decision results has become a hot topic for research. Recently Ren et al. [26] presented the normal wiggly hesitant fuzzy set (NWHFS) as an extension of HFS to explore the potential information hidden behind the original data. They assume that DMs' uncertainty can be considered a general fluctuation range based on HFE diagnostic values. Liu et al. [27] developed the normal wiggly hesitant fuzzy power Muirhead means to fully exert the strength by combining power average and Muirhead mean operators on the distance measure of the NWHFE.

To more accurately describe uncertain information, Liu et al. [28] proposed the new representation mechanism with the combination of linguistic terms set and NWHFS which resulted in a useful representation tool named normal wiggly hesitant fuzzy linguistic term set (NWHFLTS). Considering the advantages of NWHFS and TODIM, Liu and Zhang [31] defined the new distance measure of two NWHFEs and put forward an extended NWHF-TODIM method to handle the MADM problems under normal wiggly information, considering that if only membership functions represent a certain degree of the attributes, the importance of uncertainty becomes ignored. Based on the idea of Pythagorean hesitant fuzzy set, Yang et al. [29] proposed a normal wiggly Pythagorean hesitant fuzzy set (NWPaHFS) that took into account both membership and nonmembership aspects. Besides, Narayanamoorthy et al. [30] presented a normal 
wiggly dual hesitant fuzzy set (NWDHFS) as an extension of NWHFS and defined a new score function for the new fuzzy set. It can express the profound ideas of membership and nonmembership information. Liu and Zhang [32] combined the MABAC (multiattributive border approximation area comparison) with prospect theory, which considers DMs psychological behavior and proposes a new method under a normal wiggly environment for handling the complex and uncertain decision-making problems.

To facilitate a better understanding, we summarize the features and differences discussed above, which are listed in Table 1.

However, due to the increasing complexity of the fundamental issues and the uncertainty of decision-makers perception, in many circumstances, there are some difficulties for the DMs to quantify their preferences by several possible values or the behaviors of the DMs cannot be characterized by using crisp values. As HFS has a severe deficiency of information, this loss has also converted to NWHFS, leading to extreme data loss, and it should be addressed. To overcome the issue, PHFS has been used instead of HFS to minimize information loss. Therefore, according to the analysis discussed above and for the sake of overcoming the weaknesses, we shall propose a set named normal wiggly probabilistic hesitant fuzzy set (NWPHFS). The significant excellence of NWPHFS is that it could depict different attributes of a target in a single framework: possible hesitant fuzzy set, its corresponding probability, and the extracted hidden information from the original PHFS. Moreover, we propose some elementary operational laws and aggregation operators of NWPHFS to aggregate the wiggly probabilistic data. Furthermore, we establish an efficient and authentic approach to deal with MADM problems under a probabilistic environment. Finally, we apply the proposed method to the research of the environmental quality assessment. An illustrative example shows our proposed method's implementation process and demonstrates that our approach is more reliable and logical.

Here is a summary of the main contributions of this article:

(1) Considering the uncertain preferences hidden behind the original probabilistic hesitant information, we propose the normal wiggly probabilistic hesitant fuzzy set, a new extension of HFS
(2) Two new aggregation operators, the normal wiggly probabilistic hesitant fuzzy weighted averaging and the normal wiggly hesitant fuzzy weighted geometric, are put forward to conclude the rankings results of alternatives in decision-making problems

(3) We proposed a new MADM method to streamline the normal wiggly probabilistic hesitant information based on NWPHFWA and NWPHFWG operators to obtain the best alternatives

Comprehensively, the paper framework is designed in the following way: Section 2 describes the essential concepts consisting of HFS, PHFS, and NWHFS. Section 3 elaborates the NWPHFS, a new form of PHF information, the score function, operational rules, and the comparison rule of NWPHFEs. Section 4 describes the new methodology to develop the MADM problems when attributes values are expressed in NWPHFS. Section 5 explains the application stages in comparing other theories to demonstrate the feasibility and validity of the discussed method.

\section{Preliminaries}

In this section, we mainly review some basic concepts of HFS, PHFS, and NWHFS such as the operational laws, the score function, and the comparison method. Moreover, we give some examples to explain the given theories.

Definition 1 (see [8]). For a given nonempty set $S$, HFS $H$ on $S$ is a function of $h_{S}(x)$ which when applied to $S$ returns to a finite subset of $[0,1]$. Mathematically,

$$
H=\left\{\left\langle x, h_{S}(x)\right\rangle \mid x \in S\right\},
$$

where $h_{S}(x)$ is the discrete set of values from $[0,1]$ representing the possible membership degrees of the element $x \in S$, also called HFE, and for simplicity, we use $h_{S}(x)=h$. Subsequently, the score function, deviation function, and comparison rules were proposed and investigated as the basis for their calculation and application for HFEs [33].

Example 1. For any set $S=\left\{x_{1}, x_{2}, x_{3}\right\}$, let $h\left(x_{1}\right)=\{0.3,0.4,0.5\}, h\left(x_{2}\right)=\{0.2,0.3,0.5\}$, and $h\left(x_{3}\right)=$ $\{0.1,0.2\}$ be three HFEs. Then the set $H$ is called HFS and denoted as

$$
H=\left\{\left\langle x_{1},(0.3,0.4,0.5)\right\rangle,\left\langle x_{2},(0.2,0.3,0.5)\right\rangle,\left\langle x_{3},(0.1,0.2)\right\rangle\right\} .
$$

To enhance the preferences in decision-making problems, Zhu [9] extended the HFS to PHFS, defined as follows.

Definition 2. Let $S$ be any universe of discourse; then a PHFS on $S$ can be expressed by an expression

$$
H_{p}=\left\{\left\langle x, h_{p}(x)\right\rangle \mid x \in S\right\}
$$

where $h_{p}(x)=\gamma_{i}\left(p_{i}\right)$, representing the membership degree of the element $x \in S$ and $\gamma_{i}, p_{i} \in[0,1]$. For simplicity, we denote

$$
h_{p}(x)=h_{p}=\left\{\gamma_{i}\left(p_{i}\right) \mid i=1,2,3, \ldots, \# h\right\},
$$

where $\# h$ is the number of possible elements in $h_{p}, p_{i}$ is the hesitant probability of $\gamma_{i}$, and $\sum_{i=1}^{\# h} p_{i}=1$. 
TABLE 1: A summary on the normal wiggly hesitant fuzzy set and its extensions.

\begin{tabular}{|c|c|c|c|c|}
\hline Studies & $\begin{array}{c}\text { Different } \\
\text { models }\end{array}$ & Characteristic of the elements & $\begin{array}{c}\text { Extract } \\
\text { information }\end{array}$ & $\begin{array}{l}\text { Probabilistic } \\
\text { information }\end{array}$ \\
\hline [8] & HFS & A set of possible membership values & No & No \\
\hline [10] & PrHFS & A set of possible membership values and their associated proportion & No & No \\
\hline [9] & PHFS & A probabilistic distribution of several membership values & No & Yes \\
\hline [26] & NWHFS & A set of possible membership values & Yes & No \\
\hline [28] & NWHFLTS & A set of several ordered and continuous linguistic terms & Yes & No \\
\hline [29] & NWPaHFS & $\begin{array}{l}\text { A set of membership and nonmembership values such that the sum of square } \\
\text { of membership and nonmembership values is less than one }\end{array}$ & Yes & No \\
\hline$[30]$ & NWDHFS & A set of various membership and nonmembership values & Yes & No \\
\hline Proposed & NWPHFS & A probabilistic distribution of several membership values & Yes & Yes \\
\hline
\end{tabular}

2.1. Normalization of PHFEs. Usually, for the PHFS, we always hope that all the elements have complete probabilistic information. If given, then the calculation of the PHFEs will be more straightforward, and the outcome of this set-based decision-making will be more accurate. Unfortunately, the probabilistic information is not always complete. To overcome this issue, Zhang et al. [18] estimate the missing probabilistic details based on the following principle.

Definition 3. For any PHFE $h_{p}$, if $\sum_{i=1}^{\# h} p_{i}<1$, then its associated PHFE $\widehat{h}_{p}$ is defined as

$$
\widehat{h}_{p}=\left\{\gamma_{i}\left(\widehat{p}_{i}\right) \mid i=1,2,3, \ldots, \# h_{p}\right\},
$$

where $\widehat{p}_{i}=\left(p_{i} / \sum_{i=1}^{\# h} p_{i}\right)$.

To study further deeply the probable uncertainty concealed behind the assessments of DMs, Ren et al. [26] presented the concept of NWHFS.

Definition 4. For a given HFE $h=\left\{\gamma_{1}, \gamma_{2}, \gamma_{3}, \ldots, \gamma_{\# h}\right\}$, the mean value and the deviation function of all values in $h$ can be defined as

$$
\bar{h}=\frac{1}{\# h} \sum_{i=1}^{\# h} \gamma_{i}, \sigma_{h}=\sqrt{\frac{1}{\# h} \sum_{i=1}^{\# h}\left(\gamma_{i}-\bar{h}\right)^{2}},
$$

respectively. The mapping $\tilde{f}$ from $h$ to $\left[o, \sigma_{h}\right]$, which satisfies the relation,

$$
\tilde{f}\left(\gamma_{i}\right)=\sigma_{h} \cdot e^{-\left(\left(\gamma_{i}-\bar{h}\right)^{2} / 2 \sigma_{h}^{2}\right)},
$$

is said to be the normal wiggly range of $\gamma_{i}$.

Definition 5 (see [26]). Let $h=\left\{\gamma_{1}, \gamma_{2}, \gamma_{3}, \ldots, \gamma_{\# h}\right\}$ be an HFE. The normalized HFE can be calculated by the expression

$$
\begin{aligned}
& \tilde{h}=\left\{\frac{\gamma_{1}}{\operatorname{sum}\left(\gamma_{i}\right)}, \frac{\gamma_{2}}{\operatorname{sum}\left(\gamma_{i}\right)}, \frac{\gamma_{3}}{\operatorname{sum}\left(\gamma_{i}\right)}, \ldots, \frac{\gamma_{\# h}}{\operatorname{sum}\left(\gamma_{i}\right)}\right\}, \\
& \tilde{h}=\left\{\tilde{\gamma}_{1}, \tilde{\gamma}_{2}, \tilde{\gamma}_{3}, \ldots, \tilde{\gamma}_{\# h}\right\},
\end{aligned}
$$

where $\operatorname{sum}\left(\gamma_{i}\right)=\sum_{i=1}^{\# h} \gamma_{i}$. From the normalized set $\tilde{h}$, the real preference degree of DMs can be computed as follows:

$$
\operatorname{rpd}(\widetilde{h})= \begin{cases}\sum_{i=1}^{\# h} \widetilde{\gamma}_{i}\left(\frac{\# \widetilde{h}-i}{\# \widetilde{h}-1}\right), & \text { if } \bar{h}<0.5, \\ 1-\sum_{i=1}^{\# h} \widetilde{\gamma}_{i}\left(\frac{\# \widetilde{h}-i}{\# \widetilde{h}-1}\right), & \text { if } \bar{h}>0.5, \\ 0.5, & \text { if } \bar{h}=0.5,\end{cases}
$$

which is measured based on the orness, proposed by Yager [34].

Definition 6 (see [26]). For any nonempty set $S$, consider an HFE, $\quad h(x)=\left\{\gamma_{1}, \gamma_{2}, \gamma_{3}, \ldots, \gamma_{\# h}\right\}, \quad$ in an HFS, $H=\left\{\left\langle x, h_{S}(x)\right\rangle \mid x \in S\right\}$. The NWHFS can be stated as

$$
\mathrm{NW}=\left\{\left\langle x, h_{S}(x), \psi\left(h_{S}(x)\right)\right\rangle \mid x \in S\right\},
$$

where $\psi\left(h_{S}(x)\right)=\left\{\widehat{\gamma}_{1}, \widehat{\gamma}_{2}, \widehat{\gamma}_{2}, \ldots, \widehat{\gamma}_{\# h(x)}\right\}$ and

$$
\widehat{\gamma}_{i}=\left\{\tau_{i}^{L}, \tau_{i}^{M}, \tau_{i}^{U}\right\},
$$

where $\tau_{i}^{L}=\max \left(\gamma_{i}-\tilde{f}\left(\gamma_{i}\right), 0\right), \quad \tau_{i}^{M}=(2 \cdot \operatorname{rpd}(\widetilde{h}(x))-1)$ $\tilde{f}\left(\gamma_{i}\right)+\gamma_{i}$, and $\tau_{i}^{U}=\min \gamma_{i}+\widetilde{f}\left(\gamma_{i}\right), 1 . \gamma_{i}$ is one of the elements in HFE, $\tilde{f}\left(\gamma_{i}\right)$ is the wiggly parameter, and $\operatorname{rpd}(\widetilde{h}(x))$ is the real preference degree which can be found using (9). Furthermore, $\psi(h(x))$ is an NWE. For simplicity, $(h(x), \psi(h(x)))$ can be labeled as $(h, \psi(h))$, which is NWHFE.

For a better understanding, we give a simple numerical example of NWHFS. 
Example 2. Let $S=\left\{x_{1}, x_{2}, x_{3}\right\}$ and consider the HFS set $H$ to be

$$
H=\left\{\left\langle x_{1},(0.3,0.4,0.5)\right\rangle,\left\langle x_{2},(0.2,0.3,0.5)\right\rangle,\left\langle x_{3},(0.1,0.2)\right\rangle\right\} .
$$

NWHFS can be obtained according to Definitions 5 and

6:

$$
\mathrm{NW}_{H}=\left\{\begin{array}{llll}
x_{1} & (0.2614,0.2936,0.3386) & (0.3184,0.3864,0.4816) & (0.4614,0.4936,0.5386) \\
x_{2} & (0.1296,0.1789,0.2704) & (0.1797,0.2639,0.4203) & (0.4489,0.4847,0.5511) \\
x_{3} & (0.0184,0.1272,0.1816) & (0.1614,0.2129,0.2386) &
\end{array}\right\} .
$$

Some properties and operational laws for the comparison of two NWHFEs are defined as follows.

Definition 7 (see [26]). For the two NWHFEs $\left(h_{1}, \psi\left(h_{1}\right)\right)$ and $\left(h_{2}, \psi\left(h_{2}\right)\right)$, the score values can be calculated by the expression

$$
S_{\mathrm{NW}}\left(h_{1}, \psi\left(h_{1}\right)\right)=\lambda\left(\bar{h}-\sigma_{h}\right)+(1-\lambda)\left(\frac{1}{\# h} \sum_{i=1}^{\# h}\left(\overline{\widehat{\gamma}}_{i}-\tau{\widehat{\gamma_{i}}}_{i}\right)\right)
$$

where $\quad \overline{\hat{\gamma}}_{i}=\left(\left(\tau_{i}^{L}+\tau_{i}^{M}+\tau_{i}^{U}\right) / 3\right) \quad$ and $\quad \tau_{\hat{\gamma}_{i}}=$ $\sqrt{\left(\tau_{i}^{L}\right)^{2}+\left(\tau_{i}^{M}\right)^{2}+\left(\tau_{i}^{U}\right)^{2}-\tau_{i}^{L} \tau_{i}^{M}-\tau_{i}^{M} \tau_{i}^{U}-\tau_{i}^{L} \tau_{i}^{U}}$ and parameter $\lambda \in(0,1)$ is the confidence level of the DMs which can be selected according to the information given by them.

The assessment of any two NWHFEs $\left(h_{1}, \psi\left(h_{1}\right)\right)$ and $\left(h_{2}, \psi\left(h_{2}\right)\right)$ based on Definition 7 can be defined as follows:

(1) If $S_{\mathrm{NW}}\left(h_{1}, \psi\left(h_{1}\right)\right)>S_{\mathrm{NW}}\left(h_{2}, \psi\left(h_{2}\right)\right)$, then $\left(h_{1}, \psi\left(h_{1}\right)\right)$ is preferable to $\left(h_{2}, \psi\left(h_{2}\right)\right)$ and is described as $\left(h_{1}, \psi\left(h_{1}\right)\right)>\left(h_{2}, \psi\left(h_{2}\right)\right)$

(2) If $\quad S_{\mathrm{NW}}\left(h_{1}, \psi\left(h_{1}\right)\right)<S_{\mathrm{NW}}\left(h_{2}, \psi\left(h_{2}\right)\right)$, then $\left(h_{2}, \psi\left(h_{2}\right)\right)$ is preferable to $\left(h_{1}, \psi\left(h_{1}\right)\right)$ and is described as $\left(h_{1}, \psi\left(h_{1}\right)\right)<\left(h_{2}, \psi\left(h_{2}\right)\right)$

(3) If $S_{\mathrm{NW}}\left(h_{1}, \psi\left(h_{1}\right)\right)=S_{\mathrm{NW}}\left(h_{2}, \psi\left(h_{2}\right)\right)$, then both $\left(h_{1}, \psi\left(h_{1}\right)\right)$ and $\left(h_{2}, \psi\left(h_{2}\right)\right)$ are equivalent and are described as $\left(h_{1}, \psi\left(h_{1}\right)\right) \sim\left(h_{2}, \psi\left(h_{2}\right)\right)$

Definition 8 (see [26]). For any two NWHFEs $\left(h_{1}, \psi\left(h_{1}\right)\right)$ and $\left(h_{2}, \psi\left(h_{2}\right)\right)$ and $\kappa>0$, we have
(1) $\left(h_{1}, \psi\left(h_{1}\right)\right) \widehat{\oplus}\left(h_{2}, \psi\left(h_{2}\right)\right)=\left(\cup_{\gamma_{1} \in h_{1}, \gamma_{2} \in h_{2}} \gamma_{1}+\right.$ $\left.\gamma_{2}-\gamma_{1} \gamma_{2}, U_{\widehat{\gamma}_{1} \in \psi\left(h_{1}\right), \widehat{\gamma}_{2} \in \psi\left(h_{2}\right)} \widehat{\gamma}_{1} \oplus \widehat{\gamma}_{2}\right)$
(2) $\left(h_{1}, \psi\left(h_{1}\right)\right) \otimes\left(h_{2}, \psi\left(h_{2}\right)\right)=\left(U_{\gamma_{1} \in h_{1}, \gamma_{2} \in h_{2}} \gamma_{1} \gamma_{2}\right.$

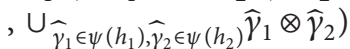
(3) $\left(h_{1}, \psi\left(h_{1}\right)\right)^{\kappa}=\left(\cup_{\gamma_{1} \in h_{1}} \gamma_{1}^{\kappa}, \cup_{\hat{\gamma}_{1} \in \psi\left(h_{1}\right.} \widehat{\gamma}_{1}^{\kappa}\right)$
(4) $\kappa\left(h_{1}, \psi\left(h_{1}\right)\right)=\left(\cup_{\gamma_{1} \in h_{1}} 1-\left(1-\gamma_{1}\right)^{\kappa}, \cup_{\hat{\gamma}_{1} \in \psi\left(h_{1}\right)} \kappa \widehat{\gamma}_{1}\right)$

\section{Normal Wiggly Probabilistic Hesitant Fuzzy Set}

Recently, Ren et al. [26] proposed the NWHFS to ensure the validity of evaluation results, which developed a methodology to dig the secret consent of DMs from the actual evaluation information. In this part, we put forward the concepts of normal wiggly parameter and real preference degree to mine the uncertain preferences hidden behind the original PHFS. Based on this, we present the NWPHFS with its operational laws and comparison method.

Definition 9. Let $h_{p}=\left\{\gamma_{1}\left(p_{1}\right), \gamma_{2}\left(p_{2}\right), \gamma_{3}\left(p_{3}\right), \ldots, \gamma_{\# h}\right.$ $\left.\left(p_{\# h}\right)\right\}$ be a PHFE. Then mean and deviation of all values in $h_{p}$ are defined as

$$
\begin{gathered}
\bar{h}_{p}=\frac{\left(\sum_{i=1}^{\# h} \gamma_{i} p_{i}\right)}{\sum_{i=1}^{\# h} p_{i}}, \\
\sigma_{h_{p}}=\frac{\sum_{i=1}^{\# h} p_{i}\left(\gamma_{i}-\bar{h}_{p}\right)^{2}}{\sum_{i=1}^{\# h} p_{i}},
\end{gathered}
$$

and the mapping $\tilde{g}: h_{p} \longrightarrow\left[0, \sigma_{h_{p}}\right]$ is defined as

$$
\tilde{g}\left(\gamma_{i}\left(p_{i}\right)\right)=\sigma_{h_{p}} e^{-0.5\left(p_{i}\left(\gamma_{i}-\bar{h}_{p}\right)^{2} /\left(\sigma_{h_{p}}\right)^{2}\right)} .
$$

Then, the interval $\left[\gamma_{i}-\tilde{g}\left(\gamma_{i}\left(p_{i}\right)\right), \gamma_{i}+\tilde{g}\left(\gamma_{i}\left(p_{i}\right)\right)\right]$ with the associated probabilistic element is called normal wiggly range of the element $\gamma_{i}\left(p_{i}\right)$.

For further clarity, an example is given in the following.

Example 3. Consider $h_{p}=\{0.2(0.1), 0.3(0.2), 0.6(0.3)\}$. The mean, deviation, and wiggle range value using Definition 9 can be calculated as 


$$
\begin{aligned}
\bar{h}_{p} & =\frac{0.2(0.1)+0.3(0.2)+0.6(0.3)}{0.1+0.2+0.3}=0.4333 \\
\sigma_{h_{p}} & =\frac{0.1(0.2-0.4333)^{2}+0.2(0.3-0.4333)^{2}+0.3(0.6-0.4333)^{2}}{0.1+0.2+0.3}=0.02889,
\end{aligned}
$$

and wiggle range corresponding to the probabilistic elements using (16) is

$$
\left\{\begin{array}{l}
0.2(0.1) \longrightarrow[0.01889,0.02111] \\
0.3(0.2) \longrightarrow[0.05657,0.06343] \\
0.6(0.3) \longrightarrow[0.1798,0.1802]
\end{array}\right.
$$

Real preference degree measured and defined by Ren et al. [26] is applicable for the HFE. For the PHFE, we extend the preference degree as follows.

Definition 10. The real preference degree of the DM in a PHFE can be calculated based on the degree of orness [34] which can be defined as

$$
\operatorname{rpd}\left(\widehat{h}_{p}\right)= \begin{cases}\sum_{i=1}^{\# h} \gamma_{i}\left(\frac{\# \widehat{h}_{p}-i}{\# \widehat{h}_{p}-1}\right) \widehat{p}_{i}, & \text { if } \bar{h}_{p}<0.5, \\ 1-\sum_{i=1}^{\# h} \gamma_{i}\left(\frac{\# \widehat{h}_{p}-i}{\# \widehat{h}_{p}-1}\right) \widehat{p}_{i}, & \text { if } \bar{h}_{p}>0.5, \\ 0.5, & \text { if } \bar{h}_{p}=0.5,\end{cases}
$$

where $\widehat{h}_{p}$ is the normalized set of $h_{p}$ calculated as

$$
\widehat{h}_{p}=\left\{\gamma_{1}\left(\widehat{p}_{1}\right), \gamma_{2}\left(p_{2}\right), \widehat{\gamma}_{3}\left(p_{3}\right), \ldots, \widehat{\gamma}_{\# h_{p}}\left(p_{\# h_{p}}\right)\right\},
$$

and $\widehat{p}_{i}=\left(p_{i} / \sum_{i=1}^{\# h} p_{i}\right),\left(i=1,2,3, \ldots, \# h_{p}\right)$.

Example

$$
\text { 4. Consider }
$$
a

PHFE $h_{p}=\{0.2(0.1), 0.3(0.2), 0.6(0.3)\}$. First the normalized set for the determination of real preference degree can be calculated. After the normalization, real preference degree can be find using equation (19) as

$$
\widehat{h}_{p}=\{0.2(0.1667), 0.3(0.3333), 0.6(0.5)\} \text {. }
$$

Then,

$$
\operatorname{rpd}\left(\widehat{h}_{p}\right)=0.08333
$$

Definition 11. Let $S=\left\{\left\langle x, h_{p}(x)\right\rangle \mid x \in S\right\}$ be a PHFS. The NWPHFS on $S$ is denoted as

$$
\mathrm{NWP}=\left\{\left\langle x, h_{p}(x), \xi\left(h_{p}(x)\right) \mid \widehat{p}\right\rangle\right\},
$$

where $h_{p}(x)$ is the PHFE, and

$$
\begin{aligned}
\xi\left(h_{p}(x)\right) \mid \widehat{p} & =\left\{\widehat{\gamma}_{1}\left(\widehat{p}_{1}\right), \hat{\gamma}_{2}\left(\widehat{p}_{2}\right), \hat{\gamma}_{3}, \ldots, \widehat{\gamma}_{\# h_{p}}\left(\widehat{p}_{\# h}\right)\right\}, \\
\hat{\gamma}_{i} & =\left\{\delta_{i}^{L}, \delta_{i}^{M}, \delta_{i}^{U}\right\}, \\
\delta_{i}^{L} & =\max \left(\gamma_{i}-\tilde{g}\left(\gamma_{i}\left(p_{i}\right)\right), 0\right), \\
\delta_{i}^{M} & =\left(2 \operatorname{rpd}\left(\widehat{h}_{p}(x)\right)-1\right) \tilde{g}\left(\gamma_{i}\left(p_{i}\right)\right)+\gamma_{i} \cdot p_{i}, \\
\delta_{i}^{U} & =\min \left(\gamma_{i}+\tilde{g}\left(\gamma_{i}\left(p_{i}\right)\right), 1\right),
\end{aligned}
$$

where $\gamma_{i}=1,2,3, \ldots, \# h_{p}$. The pair $\left\langle h_{p}(x), \xi\left(h_{p}(x)\right) \mid \widehat{p}\right\rangle$ is called NWHPHFE; for simplicity, we symbolize it as $\left\langle h_{p}, \xi\left(h_{p}\right)\right\rangle$.

For further understanding, an example is given below:

$$
\mathrm{NWP}_{H_{P}}=\left\{\begin{array}{l}
x_{1},\left(\begin{array}{l}
0.01889, \\
0.01908, \\
0.02111
\end{array}\right)\left|0.1667,\left(\begin{array}{c}
0.05657, \\
0.05714, \\
0.06343
\end{array}\right)\right| 0.3333,\left(\begin{array}{c}
0.17980, \\
0.17984, \\
0.18020
\end{array}\right) \mid 0.5, \\
x_{2}, \quad\left(\begin{array}{l}
0.05672, \\
0.06000, \\
0.06328
\end{array}\right)\left|0.2,\left(\begin{array}{c}
0.19162, \\
0.20000, \\
0.20838
\end{array}\right)\right| 0.5,\left(\begin{array}{c}
0.23999, \\
0.24000, \\
0.24001
\end{array}\right) \mid 0.3, \\
x_{3},\left(\begin{array}{l}
0.12000, \\
0.12000, \\
0.12000
\end{array}\right)\left|0.3333,\left(\begin{array}{c}
0.23841, \\
0.24074, \\
0.24159
\end{array}\right)\right| 0.4444,\left(\begin{array}{c}
0.14000, \\
0.14000, \\
0.14000
\end{array}\right) \mid 0.2222 .
\end{array}\right.
$$


Example 5. For a PHFS $H_{p}=\left\{\left\langle x_{1},(0.2(0.1), 0.3(0.2)\right.\right.$, $0.6(0.3))\rangle, \quad\left\langle x_{2},(0.3(0.2), 0.4(0.5), 0.8(0.3))\right\rangle, \quad\left\langle x_{3},(0.4\right.$ (0.3), 0.6(0.4), 0.7 (0.2)) $\rangle$ \}. Then, using Definition 11, we get an NWPHFS:

For the evaluation of NWPHF information, the score function defined below can simplify the probabilistic information into crisp values that can rationalize real-time information. As NWPHFS is in the form of the triangular fuzzy number $[35,36]$, some operations of the triangular fuzzy number are involved in finding the score of the NWPHFE.
Definition 12. Let $\left\langle h_{p}, \xi\left(h_{p}\right)\right\rangle$ be an NWPHFE; $\bar{h}_{p}$ and $\sigma_{h_{p}}$ are the mean value and deviation values of $h_{p}$. Then the score function of $\left\langle h_{p}, \xi\left(h_{p}\right)\right\rangle$ is calculated as follows:

$$
S_{\mathrm{NWP}}\left(\left\langle h_{p}, \xi\left(h_{p}\right)\right\rangle\right)=\lambda\left(\bar{h}_{p}-\sigma_{h_{p}}\right)+(1-\lambda)\left(\sum_{i=1}^{\# h}\left(\overline{\hat{\gamma}}_{i}-\tau_{\widehat{\gamma}_{i}}\right)^{2} \widehat{p}_{i}\right),
$$

where

$$
\begin{aligned}
& \overline{\hat{\gamma}}_{i}=\frac{\left(\delta_{i}^{L}+\delta_{i}^{M}+\delta_{i}^{U}\right)}{3} \cdot \widehat{p}_{i}, \\
& \tau_{\widehat{\gamma}_{i}}=\sqrt{\left(\left(\delta_{i}^{L}\right)^{2}+\left(\delta_{i}^{M}\right)^{2}+\left(\delta_{i}^{U}\right)^{2}-\delta_{i}^{L} \cdot \delta_{i}^{M}-\delta_{i}^{M} \cdot \delta_{i}^{U}-\delta_{i}^{U} \cdot \delta_{i}^{L}\right)} \cdot \widehat{p}_{i} .
\end{aligned}
$$

Here, $\lambda$ indicates the attitude and risk-bearing of the decision-makers.

(1) If the value of $\lambda$ is greater than 0.5 , then he is a risk averter

(2) If the value of $\lambda$ is less than 0.5 , then he is risk-averse

(3) If the value of $\lambda$ is 0.5 , then he is not taking any risk

The following conclusions can easily be obtained based on Definition 12.

For any two different NWPHFEs $\left\langle h_{p}^{1}, \xi\left(h_{p}^{1}\right)\right\rangle$ and $\left\langle h_{p}^{2}, \xi\left(h_{p}^{2}\right)\right\rangle$, their corresponding score values are $S_{\mathrm{NWP}}\left\langle h_{p}^{1}, \xi\left(h_{p}^{1}\right)\right\rangle$ and $S_{\mathrm{NWP}}\left\langle h_{p}^{2}, \xi\left(h_{p}^{2}\right)\right\rangle$, respectively:

(1) If $S_{\mathrm{NWP}}\left\langle h_{p}^{1}, \xi\left(h_{p}^{1}\right)\right\rangle>S_{\mathrm{NWP}}\left\langle h_{p}^{2}, \xi\left(h_{p}^{2}\right)\right\rangle$, then $\left\langle h_{p}^{1}, \xi\left(h_{p}^{1}\right)\right\rangle$ is preferable to $\left\langle h_{p}^{2}, \xi\left(h_{p}^{2}\right)\right\rangle$ and is described as $\left.\left\langle h_{p}^{1}, \xi\left(h_{p}^{1}\right)\right\rangle\right\rangle\left\langle h_{p}^{2}, \xi\left(h_{p}^{2}\right)\right\rangle$

(2) If $S_{\mathrm{NWP}}\left\langle h_{p}^{1}, \xi\left(h_{p}^{1}\right)\right\rangle<S_{\mathrm{NWP}}\left\langle h_{p}^{2}, \xi\left(h_{p}^{2}\right)\right\rangle$, then $\left\langle h_{p}^{2}, \xi\left(h_{p}^{2}\right)\right\rangle$ is preferable to $\left\langle h_{p}^{1}, \xi\left(h_{p}^{1}\right)\right\rangle$ and is described as $\left\langle h_{p}^{1}, \xi\left(h_{p}^{1}\right)\right\rangle \prec\left\langle h_{p}^{2}, \xi\left(h_{p}^{2}\right)\right\rangle$

(3) If $S_{\mathrm{NWP}}\left\langle h_{p}^{1}, \xi\left(h_{p}^{1}\right)\right\rangle=S_{\mathrm{NWP}}\left\langle h_{p}^{2}, \xi\left(h_{p}^{2}\right)\right\rangle$, then both $\left\langle h_{p}^{2}, \xi\left(h_{p}^{2}\right)\right\rangle$ and $\left\langle h_{p}^{1}, \xi\left(h_{p}^{1}\right)\right\rangle$ are equivalent and are described as $\left\langle h_{p}^{1}, \xi\left(h_{p}^{1}\right)\right\rangle \sim\left\langle h_{p}^{2}, \xi\left(h_{p}^{2}\right)\right\rangle$

For better understanding, an example is given in the following.

Example 6. The NWPHFS is taken from Example 5. Then the score value and the other values which are used to find the score are shown in Table 2 . The parameter $(\lambda=(1 / 2))$ is taken just for the simplicity which means that the DMs are neutral.

According to Definition 12, the ranking is

$$
\mathrm{NWPHFE}_{x_{3}}>\mathrm{NWPHFE}_{x_{2}}>\mathrm{NWPHFE}_{x_{1}} \text {. }
$$

3.1. Basic Operations for the NWPHFEs. Like the operational rule of HFEs [33] and NWHFEs, the following are basic rules for the operation of NWPHFEs.

(1) $\left(\left\langle h_{p}^{1}, \xi\left(h_{p}^{1}\right)\right\rangle\right)^{\lambda}=\left[\cup_{\gamma_{1_{i}} \in h_{p}^{1}}\left\{\gamma_{1_{i}}^{\lambda}\left(\widehat{p}_{1_{i}}\right)\right\}, \cup_{\widehat{\gamma}_{1_{i}} \in h_{p}^{1}}\left\{\widehat{\gamma}_{1_{i}}^{\lambda}\right.\right.$ $\left.\left.\left(\widehat{p}_{1},\right)\right\}\right]$

(2) $\lambda\left(\left\langle h_{p}^{1}, \xi\left(h_{p}^{1}\right)\right\rangle\right)=\left[\cup_{\gamma_{1_{i}} \in h_{p}^{1}}\left\{\left[1-\left(1-\gamma_{1 i}\right)^{l}\right]\left(\widehat{p}_{1_{i}}\right)\right\}\right.$, $\left.\cup \widehat{\gamma}_{1_{i}} \in h_{p}^{1}\left\{\lambda \widehat{\gamma}_{1_{i}}\left(\widehat{p}_{1_{i}}\right)\right\}\right]$

(3) $\left\langle h_{p}^{1}, \xi\left(h_{p}^{1}\right)\right\rangle \widehat{\oplus}\left\langle h_{p}^{2}, \xi\left(h_{p}^{2}\right)\right\rangle=\left[\cup_{\gamma_{1_{i}} \in h_{p}^{1}, \gamma_{2}} \in h_{p}^{2}\left\{\left[\gamma_{1_{i}}+\gamma_{2_{j}}\right.\right.\right.$

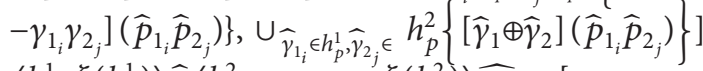

(4) $\left\langle h_{p}^{1}, \xi\left(h_{p}^{1}\right)\right\rangle \widehat{\oplus}\left\langle h_{p}^{2}, \quad \xi\left(h_{p}^{2}\right)\right\rangle \widehat{\otimes}=\left[\cup_{\gamma_{1_{i}} \in h_{p}^{1}, \gamma_{2_{j}} \in h_{p}^{2}}\right.$

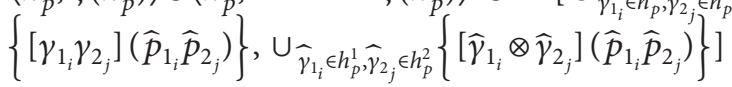

Definition 13. Let $\left\langle h_{p}^{1}, \xi\left(h_{p}^{1}\right)\right\rangle$ and $\left\langle h_{p}^{2}, \xi\left(h_{p}^{2}\right)\right\rangle$ be two NWPHFEs and $\lambda>0$; then

From the operational rules proposed above, we can see that the results are also NWPHFEs.

Theorem 1. Let $\left\langle h_{p}^{1}, \xi\left(h_{p}^{1}\right)\right\rangle,\left\langle h_{p}^{2}, \xi\left(h_{p}^{2}\right)\right\rangle$, and $\left\langle h_{p}^{3}, \xi\left(h_{p}^{3}\right)\right\rangle$ be three NWPHFEs; $\lambda>0, \lambda_{1}>0, \lambda_{2}>0$, and then

(1) $\left\langle h_{p}^{1}, \xi\left(h_{p}^{1}\right)\right\rangle \widehat{\oplus}\left\langle h_{p}^{2}, \xi\left(h_{p}^{2}\right)\right\rangle=\left(\left\langle h_{p}^{2}, \xi\left(h_{p}^{2}\right)\right\rangle\right) \widehat{\oplus}$ $\left(\left\langle h_{p}^{1}, \xi\left(h_{p}^{1}\right)\right\rangle\right)$

(2) $\left\langle h_{p}^{1}, \xi\left(h_{p}^{1}\right)\right\rangle \widehat{\oplus}\left(\left\langle h_{p}^{2}, \xi\left(h_{p}^{2}\right)\right\rangle \quad \widehat{\oplus}\left\langle h_{p}^{3}, \xi\left(h_{p}^{3}\right)\right\rangle\right)=\left(\left\langle h_{p}^{1}\right.\right.$, $\left.\left.\xi\left(h_{p}^{1}\right)\right\rangle \widehat{\oplus}\left\langle h_{p}^{2}, \xi\left(h_{p}^{2}\right)\right\rangle\right) \widehat{\oplus}\left\langle h_{p}^{3}, \xi\left(h_{p}^{3}\right)\right\rangle$

(3) $\lambda\left(\left\langle h_{p}^{1}, \xi\left(h_{p}^{1}\right)\right\rangle \widehat{\oplus}\left\langle h_{p}^{2}, \xi\left(h_{p}^{2}\right)\right\rangle\right)=\lambda\left\langle h_{p}^{1}, \xi\left(h_{p}^{1}\right)\right\rangle \widehat{\oplus} \lambda\left\langle h_{p}^{2}\right.$, $\left.\xi\left(h_{p}^{2}\right)\right\rangle$

(4) $\left(\left\langle h_{p}^{1}, \xi\left(h_{p}^{1}\right)\right\rangle \widehat{\oplus}\left\langle h_{p}^{2}, \xi\left(h_{p}^{2}\right)\right\rangle\right)^{\lambda}=\quad\left(\left\langle h_{p}^{1}, \xi\left(h_{p}^{1}\right)\right\rangle\right)^{\lambda} \widehat{\otimes}$ $\left(\left\langle h_{p}^{2}, \xi\left(h_{p}^{2}\right)\right\rangle\right)^{\lambda}$

(5) $\left(\left(\left\langle h_{p}^{1}, \xi\left(h_{p}^{1}\right)\right\rangle\right)^{\lambda_{1}}\right)^{\lambda_{2}}=\left(\left\langle h_{p}^{1}, \xi\left(h_{p}^{1}\right)\right\rangle\right)^{\lambda_{1} \lambda_{2}}$ 
TABLE 2: Mean value and deviation value of PHFEs.

\begin{tabular}{|c|c|c|c|c|c|c|c|c|c|}
\hline & $\bar{h}_{p}$ & $\sigma_{h_{p}}$ & & $\overline{\widehat{\gamma}}_{i}$ & & & $\tau_{\hat{\gamma}_{i}}$ & & $S_{\mathrm{NWP}}$ \\
\hline$x_{1}$ & 0.4333 & 0.02889 & 0.00328 & 0.01968 & 0.0900 & 0.0004 & 0.0022 & 0.0002 & 0.2043 \\
\hline$x_{2}$ & 0.5 & 0.04 & 0.01200 & 0.10000 & 0.0720 & 0.0011 & 0.0073 & 0.0000 & 0.2329 \\
\hline$x_{3}$ & 0.5556 & 0.01358 & 0.04 & 0.10678 & 0.0311 & 0.0000 & 0.0013 & 0.0000 & 0.2738 \\
\hline
\end{tabular}

Proof

(1) $\left\langle h_{p}^{1}, \xi\left(h_{p}^{1}\right)\right\rangle \widehat{\oplus}\left\langle h_{p}^{2}, \xi\left(h_{p}^{2}\right)\right\rangle=\left[\cup_{\gamma_{1_{i}} \in h_{p}^{1}, \gamma_{h_{j}} \in} h_{p}^{2}\left\{\left[\gamma_{1_{i}}\right.\right.\right.$ $\left.\left.+\gamma_{2_{j}}-\gamma_{1_{i}} \quad \gamma_{2_{j}}\right]\left(p_{1_{i}} p_{2_{j}} / \sum_{i=1}^{n^{2} j} p_{1_{i}} \sum_{i=1}^{n} p_{2_{j}}\right)\right\}$, $\left.\mathrm{U}_{\widehat{\gamma}_{1_{i}} \in h_{p}^{1}, \widehat{\gamma}_{2_{j}} \in h_{p}^{2}}\left\{\left[\widehat{\gamma}_{1} \oplus \widehat{\gamma}_{2}\right]\left(p_{1_{i}} p_{2_{j}} / \quad \sum_{i=1}^{n} p_{1_{i}} \sum_{i=1}^{n} p_{2_{j}}\right)\right\}\right]$ $=\left[U_{\gamma_{1_{i}} \in h_{p}^{1}, \gamma_{2_{j}} \in h_{p}^{2}}\left\{\left[\gamma_{2_{i}}+\gamma_{1_{j}}-\gamma_{2_{i}} \gamma_{1_{j}}\right] \quad\left(\widehat{p}_{1_{i}} \widehat{p}_{2_{j}}\right)\right\}\right.$, $\left.\mathrm{U}_{\widehat{\gamma}_{1_{i}} \in h_{p}^{1}, \widehat{\gamma}_{2_{j}} \in h_{p}^{2}}\left\{\left[\widehat{\gamma}_{2} \oplus \widehat{\gamma}_{1}\right]\left(\widehat{p}_{1_{i}} \widehat{p}_{2_{j}}\right)\right\}\right] \quad=\left\langle h_{p}^{2}, \xi\left(h_{p}^{2}\right)\right\rangle \widehat{\oplus}$ $\left\langle h_{p}^{1}, \xi\left(h_{p}^{1}\right)\right\rangle$

(2) Obvious as (1)

(3) $\lambda\left(\left\langle h_{p}^{1}, \xi\left(h_{p}^{1}\right)\right\rangle \widehat{\oplus}\left\langle h_{p}^{2}, \xi\left(h_{p}^{2}\right)\right\rangle\right)=\lambda\left[\cup_{\gamma_{1_{i}} \in h_{p}^{1}, \gamma_{2_{j}} \in h_{p}^{2}}\left\{\left[\gamma_{1_{i}}\right.\right.\right.$ $\left.\left.+\gamma_{2_{j}}-\gamma_{1_{i}} \gamma_{2_{j}}\right]\left(\widehat{p}_{1_{i}} \widehat{p}_{2_{j}}\right)\right\}, \mathrm{U}_{\widehat{\gamma}_{1_{i}} \in h_{p}^{1}, \widehat{\gamma}_{2_{j}} \in h_{p}^{2}}$

$\left.\left\{\left[\widehat{\gamma}_{1} \oplus \widehat{\gamma}_{2}\right]\left(\widehat{p}_{1_{i}} \widehat{p}_{2_{j}}\right)\right\}\right]=\quad\left[\cup_{\gamma_{1_{i}} \in h_{p}^{1}, \gamma_{2_{j}} \in h_{p}^{2}} \quad\{(1-(1-\right.$ $\left.\left.\left.\left(\gamma_{1_{i}}+\gamma_{2_{j}}-\gamma_{1_{i}} \quad \gamma_{2_{j}}\right)^{\lambda}\right)\right)\left(\widehat{p}_{1_{i}} \widehat{p}_{2_{j}}\right)\right\}, \cup_{\hat{\gamma}_{1_{i}} \in h_{p}^{1}, \widehat{\gamma}_{2_{j}} \in h_{p}^{2}}$ $\left.\left\{\lambda\left(\widehat{\gamma}_{1} \oplus \widehat{\gamma}_{2}\right)\left(\widehat{p}_{1_{i}} \widehat{p}_{2_{j}}\right)\right\}\right]=\left[\cup_{\gamma_{1_{i}} \in h_{p}^{1}, \gamma_{2} \in h_{p}^{2}} \quad\{[1-(1-\right.$ $\left(1-\left(1-\gamma_{1_{i}}\right)\left(1-\gamma_{2_{j}}\right)\right)$

$\left.\left.\lambda)]\left(\widehat{p}_{1_{i}} \widehat{p}_{2_{j}}\right)\right\}, \cup \widehat{\gamma}_{1_{i}} \in h_{p}^{1}, \widehat{\gamma}_{2_{j}} \in h_{p}^{2}\left\{\left(\lambda \widehat{\gamma}_{1} \oplus \lambda \widehat{\gamma}_{2}\right)\left(\widehat{p}_{1_{i}} \widehat{p}_{2_{j}}\right)\right\}\right]=$ $\left[\cup_{\gamma_{1_{i}} \in h_{p}^{1}, \gamma_{2_{j}} \in h_{p}^{2}}\left\{\left[1-\left(1-\gamma_{1_{i}}\right)^{\lambda}+1-\left(1-\gamma_{2_{j}}\right)^{\lambda}-(1-\right.\right.\right.$ $\left.\left.\left.\left(1-\gamma_{1_{i}}\right)^{\lambda}\right)\left(1-\left(1-\gamma_{2_{j}}\right)^{\lambda}\right)\right]\left(\widehat{p}_{1_{i}} \widehat{p}_{2_{j}}\right)\right\}$

$\left.\mathrm{U}_{\widehat{\gamma}_{1_{i}} \in h_{p}^{1}, \widehat{\gamma}_{2_{j}} \in h_{p}^{2}}\left\{\left(\lambda \widehat{\gamma}_{1} \oplus \lambda \widehat{\gamma}_{2}\right)\left(\widehat{p}_{1_{i}} \widehat{p}_{2_{j}}\right)\right\}\right]=\lambda\left\langle h_{p}^{1}\right.$, $\left.\xi\left(h_{p}^{1}\right)\right\rangle \widehat{\oplus} \lambda\left\langle h_{p}^{2}, \xi\left(h_{p}^{2}\right)\right\rangle$

(4) $\left(\left\langle h_{p}^{1}, \xi\left(h_{p}^{1}\right)\right\rangle \widehat{\otimes}\left\langle h_{p}^{2}, \xi\left(h_{p}^{2}\right)\right\rangle\right)^{\lambda}=$ $\left[\cup_{\gamma_{1_{i}} \in h_{p}^{1}, \gamma_{2_{j}} \in h_{p}^{2}}\left\{\gamma_{1_{i}} \gamma_{2_{j}}\left(\widehat{p}_{1_{i}} \widehat{p}_{2_{j}}\right)\right\}, \cup_{\widehat{\gamma}_{1_{i}} \in h_{p}^{1}, \widehat{\gamma}_{2_{j}} \in h_{p}^{2}}\left\{\widehat{\gamma}_{1_{i}}^{\lambda}\right.\right.$ $\left.\left.\widehat{\gamma}_{2_{j}}^{\lambda}\left(\widehat{p}_{1_{i}} \widehat{p}_{2_{j}}\right)\right\}\right]=\left[\cup_{\gamma_{1_{i}} \in h_{p}^{1}, \gamma_{2_{j}} \in h_{p}^{2}}\left\{\gamma_{1_{i}}^{\lambda}\right.\right.$ $\left.\left.\gamma_{2_{j}}^{\lambda}\left(\widehat{p}_{1_{i}} \widehat{p}_{2_{j}}\right)\right\}, \cup \widehat{\gamma}_{1_{i}} \in h_{p}^{1}, \widehat{\gamma}_{2_{j}} \in h_{p}^{2}\left\{\widehat{\gamma}_{1_{i}}^{\lambda} \widehat{\gamma}_{2_{j}}^{\lambda}\left(\widehat{p}_{1_{i}} \widehat{p}_{2_{j}}\right)\right\}\right]=$ $\left(\left\langle h_{p}^{1}, \xi\left(h_{p}^{1}\right)\right\rangle\right)^{\lambda} \widehat{\otimes}\left(\left\langle h_{p}^{2}, \xi\left(h_{p}^{2}\right)\right\rangle\right)^{\lambda}$

(5) $\left(\left(\left\langle h_{p}^{1}, \xi\left(h_{p}^{1}\right)\right\rangle\right)^{\lambda_{1}}\right)^{\lambda_{2}}=$ $\left[\cup_{\gamma_{1_{i}} \in h_{p}^{1}}\left\{\gamma_{1_{i}}^{\lambda_{1}}\left(\widehat{p}_{1_{i}}\right)\right\}, \cup_{\widehat{\gamma}_{1_{i}} \in h_{p}^{1}}\left\{\widehat{\gamma}_{1_{i}}^{\lambda_{1}}\left(\widehat{p}_{1_{i}}\right)\right\}\right]$

$\lambda_{2}=\left[\cup_{\gamma_{1_{i}} \in h_{p}^{1}}\left\{\gamma_{1_{i}}^{\lambda_{1} \lambda_{2}}\left(\widehat{p}_{1_{i}}\right)\right\}\right.$,

$\left.\cup_{\widehat{\gamma}_{1_{i}} \in h_{p}^{1}}\left\{\widehat{\gamma}_{1_{i}} \lambda_{1} \lambda_{2}\left(\widehat{p}_{1_{i}}\right)\right\}\right]=\left(\left\langle h_{p}^{1}, \xi\left(h_{p}^{1}\right)\right\rangle\right)^{\lambda_{1} \lambda_{2}}$

3.2. Aggregation Operators for the NWPHFEs. Aggregation operators for the NWPHFS depend upon the properties given in Section 3.1. These operators are very suitable and significant to handle the MADM problems with NWPHF information.

$$
\begin{aligned}
& \operatorname{NWPHFWA}\left(\left\{\left\langle h_{p}^{i}, \xi\left(h_{p}^{i}\right)\right\rangle \mid i=1,2,3, \ldots, n\right\}\right)=\widehat{\oplus}_{i=1}^{n} \omega_{i}\left(\left\langle h_{p}^{i}, \xi\left(h_{p}^{i}\right)\right\rangle\right) \\
& \left.=\left\{\underset{\gamma_{i} \in h_{p}^{i}}{\cup}\left(1-\prod_{i=1}^{n}\left(1-\gamma_{i}\right)^{\omega_{i}}\right)\left(\frac{\prod_{i=1}^{n} p_{i}}{\prod_{i=1}^{n}\left(\sum_{i=1}^{n} p_{i}\right)}\right), \underset{\widehat{\gamma}_{i} \in h_{p}^{i}}{\cup} \underset{i=1}{\stackrel{\oplus}{\oplus} \omega_{i}}\left(\widehat{\gamma}_{i}\right)\right)\left(\frac{\prod_{i=1}^{n} p_{i}}{\prod_{i=1}^{n}\left(\sum_{i=1}^{n} p_{i}\right)}\right)\right\} .
\end{aligned}
$$

Definition 14. Consider any NWPHFS, $\mathrm{NWP}=\left\{\left\langle h_{p}^{i}, \xi\left(h_{p}^{i}\right)\right\rangle \mid i=1,2,3, \ldots, n\right\}$, a collection of NWPHFEs and let $\omega=\left(\omega_{1}, \omega_{2}, \omega_{3}, \ldots, \omega_{n}\right)$ be the weight vectors of $\left\langle h_{p}^{i}, \xi\left(h_{p}^{i}\right)\right\rangle$ with $\omega_{i} \in[0,1]$ and $\sum_{i=1}^{n} \omega_{i}=1$. Then, an NWPHF weighted averaging (NWPHFWA) operator is defined as follows:
If $\omega=((1 / n),(1 / n),(1 / n), \ldots,(1 / n))$, then $(29)$ reduces to NWPHFA operator. The NWPHFWA operator helps solve MADM problems, and its practical application is shown in Section 5.

$$
\begin{aligned}
& \operatorname{NWPHFWG}\left(\left\{\left\langle h_{p}^{i}, \xi\left(h_{p}^{i}\right)\right\rangle \mid i=1,2,3, \ldots, n\right\}\right)=\widehat{\otimes}_{i=1}^{n}\left(\left\langle h_{p}^{i}, \xi\left(h_{p}^{i}\right)\right\rangle\right)^{\omega_{i}} \\
& =\left\{\cup_{\gamma_{i} \in h_{p}^{i}}\left(\prod_{i=1}^{n} \gamma_{i}^{\omega_{i}}\right)\left(\frac{\prod_{i=1}^{n} p_{i}}{\prod_{i=1}^{n}\left(\sum_{i=1}^{n} p_{i}\right)}\right), \cup_{\widehat{\gamma}_{i} \in h_{p}^{i}}\left(\bigotimes_{i=1}^{n}\left(\widehat{\gamma}_{i}\right)^{\omega_{i}}\right)\left(\frac{\prod_{i=1}^{n} p_{i}}{\prod_{i=1}^{n}\left(\sum_{i=1}^{n} p_{i}\right)}\right)\right\} .
\end{aligned}
$$


Definition 15. Consider any NWPHFS, $\mathrm{NWP}=\left\{\left\langle h_{p}^{i}, \xi\left(h_{p}^{i}\right)\right\rangle \mid i=1,2,3, \ldots, n\right\}$, a collection of NWPHFEs and let $\omega=\left(\omega_{1}, \omega_{2}, \omega_{3}, \ldots, \omega_{n}\right)$ be the weight vectors of $\left\langle h_{p}^{i}, \xi\left(h_{p}^{i}\right)\right\rangle$ with $\omega_{i} \in[0,1]$ and $\sum_{i=1}^{n} \omega_{i}=1$. Then, an NWPHF weighted geometric (NWPHFWG) operator is defined as follows:

If $\omega=((1 / n),(1 / n),(1 / n), \ldots,(1 / n))$, then $(30)$ reduces to NWPHFG operator.

Note that, in the definition above, the weight vectors must satisfy the condition $\sum_{i=1}^{n} \omega_{i}=1$. This is per our habits and makes it easy for aggregation operators to be used. But this does not happen in most practical applications; most of the time, the situation is not in our favour, and $\sum_{i=1}^{n} \omega_{i}<1$, which is not reasonable. The issue needs to be resolved, but, fortunately, it is not a major issue. We can normalize the weight vector, and then the new weight vector satisfies the property in which it holds most of the original information.

\section{MADM Process with the NWPHFS}

In this section, we shall propose a novel approach to MADM problems with the normal wiggly probabilistic hesitant fuzzy numbers based on the NWPHFWA and NWPHFWG operators.

4.1. Proposed Methodology under the Normal Wiggly Probabilistic Hesitant Fuzzy Environment. Consider a problem having $m$ alternatives, denoted by $A=\left\{A_{1}, A_{2}, A_{3}, \ldots, A_{m}\right\}$. Each alternative is assessed based on $n$ attribute, shown by $C=\left\{C_{1}, C_{2}, C_{3}, \ldots, C_{n}\right\}$, which are weighted according to the attribute weight vector $w=\left\{w_{1}, w_{2}, w_{3}, \ldots, w_{n}\right\}$ and weights should satisfy the conditions $w_{i} \in[0,1]$ and $\sum_{i=1}^{n} \omega_{i}=1$. Finally, for the evaluation of alternatives, some experts/DMs are invited to provide the data in the form of PHFEs.

In the following, the proposed method under wiggly probabilistic environment is applied to solve such MADM problems. The steps are as follows:

Step 1. Construct a decision matrix by using PHF information given by the DMs as shown in Table 3

Step 2. According to Definition 11, another decision matrix is obtained based on NWPHFS given in Table 4

Step 3. Utilize (29) or (30) to calculate the unified assessment value of each alternative

Step 4. For the collective results, use Definition 12 to find the scores of the alternatives by simple calculation and arrange the alternatives according to assessment values with the given comparison method

To demonstrate the process of the proposed method based on NWPHF information, a flowchart is drawn as shown in Figure 1.

We have developed the concept of NWPHFS to link probabilistic information to NWHFS to minimize information loss. NWPHFS can better deal with practical problems when DMs provide their preference values based on a random variable. To address the real issues of MADM, we have come up with an appropriate approach based on NWPHF weighted aggregation operators. It can improve the diagnostic results and handle the complex information under the wiggly probabilistic environment. In addition, this technique will be applied to environmental quality testing in the next section.

\section{Application to the Environmental Quality Evaluation}

The quality of the environment plays a significant role in human life and directly impacts human health. So people are always worried about environmental degradation and make efforts to alleviate the quality of the environment. Numerous firms plan ecological projects, specifically for the chemical industry. Therefore, environmental quality assessment has a direct impact on economic and social development. It is unbearable to disregard all businesses that can contaminate the atmosphere. For sustainable development, we must find a stable point. One possible way is to assess the ecological superiority of some diverse locations and develop environmental standards for the worst spots. After that, we can have an overall ecological standard. Therefore, the real problem is to identify an area that has a bad atmosphere between different places. A comprehensive approach is proposed for the decisionmaking process as follows.

The quality of the environment depends upon the region according to certain standards and assessment procedures. On the contrary, suppose that the department of environmental protection's survey shows the four areas that need to be amended. Keeping in mind the time and cost, it is beneficial to focus all the resources in a single area. The main problem is to select one of the four areas that need to be considered first. These four areas can be described as $A_{1}, A_{2}, A_{3}$, and $A_{4}$. There are many characteristics in environmental structure, but, for illustration, we consider only four of them in this article: atmospheric environment $\left(C_{1}\right)$, water environment $\left(C_{2}\right)$, noise $\left(C_{3}\right)$, and waste material $\left(C_{4}\right)$. We provide a detailed explanation of these four attributes in Table 5. According to many environmentalists, the weight of the four attribute is given as $W=(0.3,0.25,0.2$, 0.25) and consists of several attributes. But, in this article, DM holistically considers each criterion to demonstrate the preferred information for each alternative in the form of PHFS. The combined information of the DMs based on PHFEs is shown in Table 6. As stated in Definition 9, we use the NWPHFS to drive all PHF information; Tables [6-9] can then be created for the NWPHF decision matrix.

Below is a summary of the concrete decision-making process:

Step 1. Identify the problem, a combination of each alternative $\left(A_{1}, A_{2}, A_{3}, A_{4}\right)$, set of attributes $\left(C_{1}, C_{2}, C_{3}, C_{4}\right)$, and their weight vectors $w=(0.3,0.25,0.2,0.25)$.

Step 2. Unite experts to evaluate the alternatives under attribute, and build a PHF decision matrix as shown in Table 6. 
TABle 3: Probabilistic hesitant fuzzy decision matrix given by the experts.

\begin{tabular}{cccccc}
\hline & $C_{1}$ & $C_{2}$ & $C_{3}$ & $\cdots$ & \\
\hline$A_{1}$ & $h_{11}\left(p_{11}\right)$ & $h_{12}\left(p_{12}\right)$ & $h_{13}\left(p_{13}\right)$ & $C_{n}$ \\
$A_{2}$ & $h_{21}\left(p_{21}\right)$ & $h_{22}\left(p_{22}\right)$ & $h_{23}\left(p_{23}\right)$ & $\cdots$ & \\
$A_{3}$ & $h_{31}\left(p_{31}\right)$ & $h_{32}\left(p_{32}\right)$ & $h_{33}\left(p_{33}\right)$ & $\cdots$ & $h_{1 n}\left(p_{1 n}\right)$ \\
$\vdots$ & $\vdots$ & $\vdots$ & $\vdots$ & $\cdots$ & $h_{2 n}\left(p_{2 n}\right)$ \\
$A_{m}$ & $h_{m 1}\left(p_{m 1}\right)$ & $h_{m 2}\left(p_{m 2}\right)$ & $h_{m 3}\left(p_{m 3}\right)$ & $\vdots$ & \\
\hline
\end{tabular}

TABle 4: Normal probabilistic hesitant fuzzy decision matrix.

\begin{tabular}{cccccc}
\hline & $C_{1}$ & $C_{2}$ & $C_{3}$ & $\cdots$ & \\
\hline$A_{1}$ & $\left\langle h_{p}^{11}, \xi\left(h_{p}^{11}\right)\right\rangle$ & $\left\langle h_{p}^{12}, \xi\left(h_{p}^{12}\right)\right\rangle$ & $\left\langle h_{p}^{13}, \xi\left(h_{p}^{13}\right)\right\rangle$ & $\cdots$ & \\
$A_{2}$ & $\left\langle h_{p}^{21}, \xi\left(h_{p}^{21}\right)\right\rangle$ & $\left\langle h_{p}^{22}, \xi\left(h_{p}^{22}\right)\right\rangle$ & $\left\langle h_{p}^{23}, \xi\left(h_{p}^{23}\right)\right\rangle$ & $\cdots$ & $\left\langle h_{p}^{n 1}, \xi\left(h_{p}^{n 1}\right)\right\rangle$ \\
$A_{3}$ & $\left\langle h_{p}^{31}, \xi\left(h_{p}^{31}\right)\right\rangle$ & $\left\langle h_{p}^{32}, \xi\left(h_{p}^{32}\right)\right\rangle$ & $\left\langle h_{p}^{33}, \xi\left(h_{p}^{33}\right)\right\rangle$ & $\vdots$ & $\cdots$ \\
$\vdots$ & $\vdots$ & $\vdots$ & $\vdots$ & $\vdots$ & $\left\langle h_{p}^{n 2}, \xi\left(h_{p}^{n 2}\right)\right\rangle$ \\
$A_{m}$ & $\left\langle h_{p}^{m 1}, \xi\left(h_{p}^{m 1}\right)\right\rangle$ & $\left\langle h_{p}^{m 2}, \xi\left(h_{p}^{m 2}\right)\right\rangle$ & $\left\langle h_{p}^{m 3}, \xi\left(h_{p}^{m 3}\right)\right\rangle$ & $\vdots$ & $\cdots$ \\
\hline
\end{tabular}

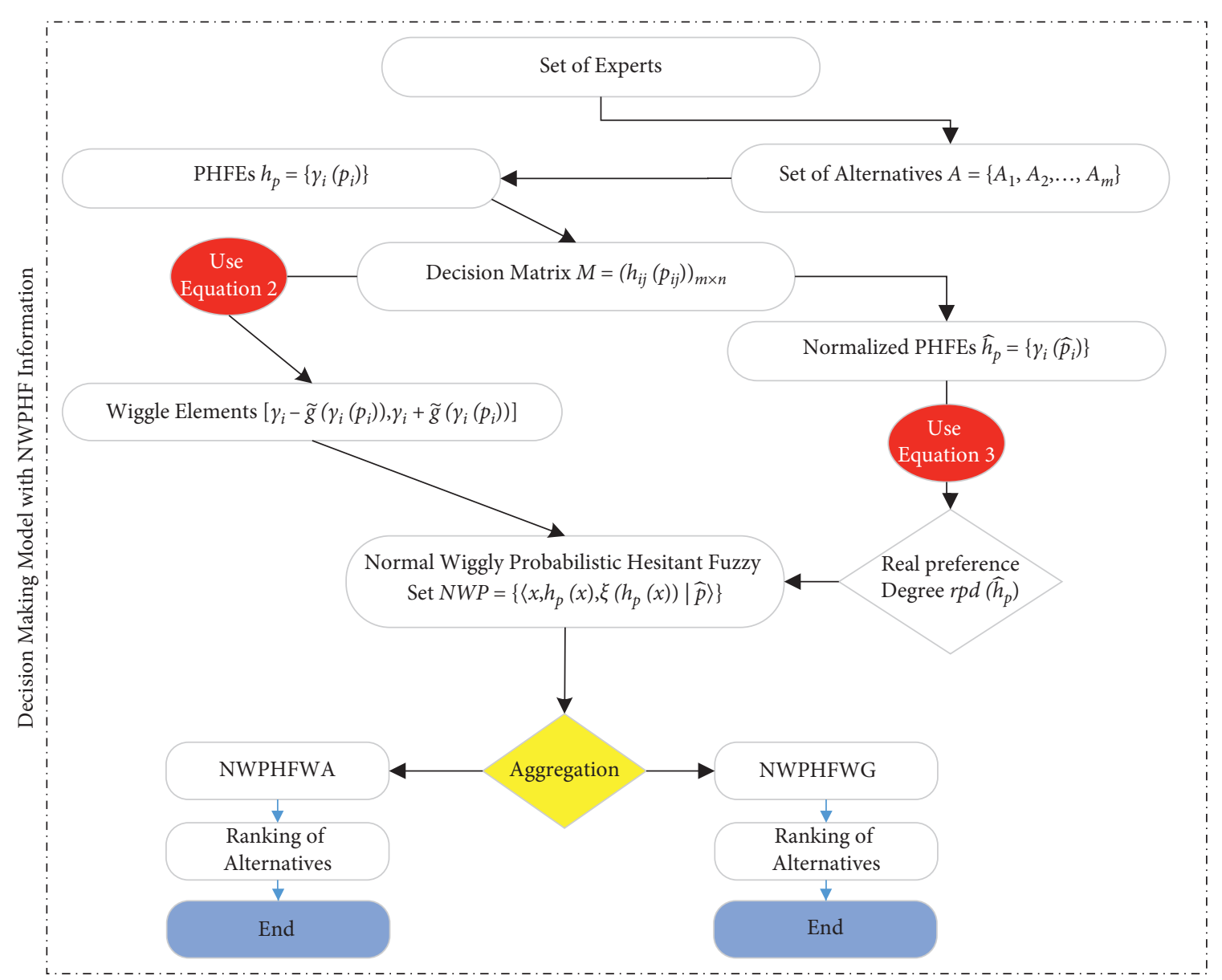

FIgURE 1: The schema of the whole decision-making steps using the normal wiggly probabilistic hesitant fuzzy information.

Step 3. NWPHF decision matrix according to Definition 9 shown in Table 7.

Step 4. Compute the combined evaluation values of each alternative by using the operators NWPHFWG and NWPHFWA, given in Definitions 14 and 15. Calculate the score values according to Definition 12, and rate all the alternatives according to their scores. The scores for the alternatives and final ranking are shown in Table 8.

However, if we seize the probabilities, the PHFEs in Table 6 will convert to HFEs. Then, using the NWHFS 
TABle 5: The description of the attributes under consideration.

\begin{tabular}{|c|c|}
\hline Attribute & Explanation \\
\hline $\begin{array}{l}C_{1}: \text { atmospheric } \\
\text { environment }\end{array}$ & Controlling air pollution and limiting greenhouse gas emissions \\
\hline$C_{2}$ : water environment & $\begin{array}{l}\text { Controlling regional irrigation pollution, guiding the effective use of aquatic resources, maintaining and } \\
\text { improving water quality and the aquatic environment, ensuring the availability of adequate water resources }\end{array}$ \\
\hline$C_{3}:$ noise & $\begin{array}{c}\text { To control noise and noise level of enclosed enclosures on urban traffic arterials, ensure the sound quality in } \\
\text { sensitive locations, such as residential areas }\end{array}$ \\
\hline$C_{4}$ : waste material & To improve the construction and nature of solid waste \\
\hline
\end{tabular}

Table 6: Probabilistic hesitant fuzzy decision matrix given by the experts.

\begin{tabular}{ccccc}
\hline & $C_{1}$ & $C_{2}$ & $C_{3}$ & $C_{4}$ \\
\hline$A_{1}$ & $\{0.15(0.3), 0.35(0.5), 0.65(0.2)\}$ & $\{0.25(0.4), 0.65(0.6)\}$ & $\{0.4(0.3), 0.8(0.7)\}$ & $\{0.1(0.3), 0.4(0.3), 0.6(0.4)\}$ \\
$A_{2}$ & $\{0.35(0.5), 0.8(0.5)\}$ & $\{0.05(0.3), 0.35(0.6), 0.75(0.1)\}$ & $\{0.25(0.4), 0.7(0.6)\}$ & $\{0.3(0.4), 0.6(0.3), 0.1(0.3)\}$ \\
$A_{3}$ & $\{0.25(0.35), 0.75(0.65)\}$ & $\{0.25(0.3), 0.65(0.5), 0.8(0.2)\}$ & $\{0.3(0.55), 0.85(0.45)\}$ & $\{0.2(0.25), 0.4(0.4), 0.7(0.35)\}$ \\
$A_{4}$ & $\{0.75(0.4), 0.55(0.3), 0.1(0.3)\}$ & $\{0.25(0.4), 0.7(0.6)\}$ & $\{0.15(0.2), 0.4(0.4), 0.85(0.4)\}$ & $\{0.65(0.4), 0.75(0.2), 0.85(0.4)\}$ \\
\hline
\end{tabular}

aggregation operators to calculate the score values of the alternatives, the score and raking can be found in Table 9.

The final ranking can be seen in Tables 8 and 9 which are different; these operators present different results, which will be discussed in the following subsection. Table 8 provides the results of NWPHFWG and NWPHFWA operators, and the result obtained by the method is given in [26] and can be obtained in Table 9. The results in Table 9 are not consistent because there is a severe loss of information. Uncertain information is dug to get more analytical results, but exploring the PHF information will provide more accurate and consistent results.

5.1. Comparative Analysis. Ren at al. [26] presented a MADM method based on two operators, namely, the NWHFWA and the NWHFWG, and applied them to evaluate alternatives under the normal wiggly hesitant fuzzy environment. In this section, we compare the proposed method with this approach to categorize the alternatives by calculating the final scores. NWPHFS can be seen as an extension of PHFS, which develops a technique for digging up potential information. In PHFSs, the DMs provide their assessment by a finite set of values along with their respective probabilities, which can better articulate their hesitation. Therefore, it is essential to compare the results of their classification. The original PHF information values are presented in Table 6. As NWHFS has a special case of NWPHFS, where their probabilities of each membership degrees are equal to one, the form NWPHFS is more general, which can help DMs express evaluation information. Since no such other procedure has been developed for NWPHFS, we compare our proposed procedure with the special case of NWPHFS, which is NWHFS. HFS can be obtained by seizing the probability in PHFS from Table 6. By using the method based on NWHFWA and NWHFWG operators, we calculate the ranking outcomes in Table 9. Table 9 indicates that the results of Ren et al. [26] based on NWHFWA and NWHFWG are not consistent, when compared to the results of our method given in Table 8 . From Table 9, it can easily be seen that $A_{3}$ is the best choice and $A_{2}$ is the worst choice; however, $A_{4}$ is the more appropriate alternative, and $A_{2}$ is the worst by NWPHFWG operator, and the ranking order of the remaining options is also different. Moreover, by utilizing the NWHFWA operator, we see that $A_{4}$ is the best choice, and $A_{1}$ is the worst choice; but $A_{4}$ is the more appropriate alternative, and $A_{2}$ is the worst by NWPHFWA operator. The main reason for the differences is that our method takes both the original hesitant information and probabilistic information into account. Furthermore, under the NWHFWA and NWHFWG operators, the rankings are different from that of our method. NWPHFSs allow DMs to express their values in membership values along with their respective probabilities more flexibly. Finally, these basically consistent ranking results demonstrate the feasibility and effectiveness of our method. Also, we easily see that if we cease the probabilities, then the proposed method and the method defined by Ren et al. [26] are the same. This also guarantees that the proposed method can handle more complex information and more space in decision-making.

5.2. Advantages of the Proposed Approach. Some advantages have been pointed out from the proposed studies concerning the present:

(1) Because the PHF set is an extension of HFS and contains more information than HFS, the proposed aggregation operators (NWPHFWA and NWPHFWG) generalize the NWHFWA and NWHFWG operators. Hence, these operators can address the decision-making difficulties more efficiently.

(2) Tables 8 and 9 show that the final results of our proposed procedure do not conform to current practices under the hesitant fuzzy environment. It is also shown that conventional HFS has a severe loss of information. Thus, a comparative study reveals that the proposed measure is more appropriate and practically workable and provides a better way under the PHF environment. 


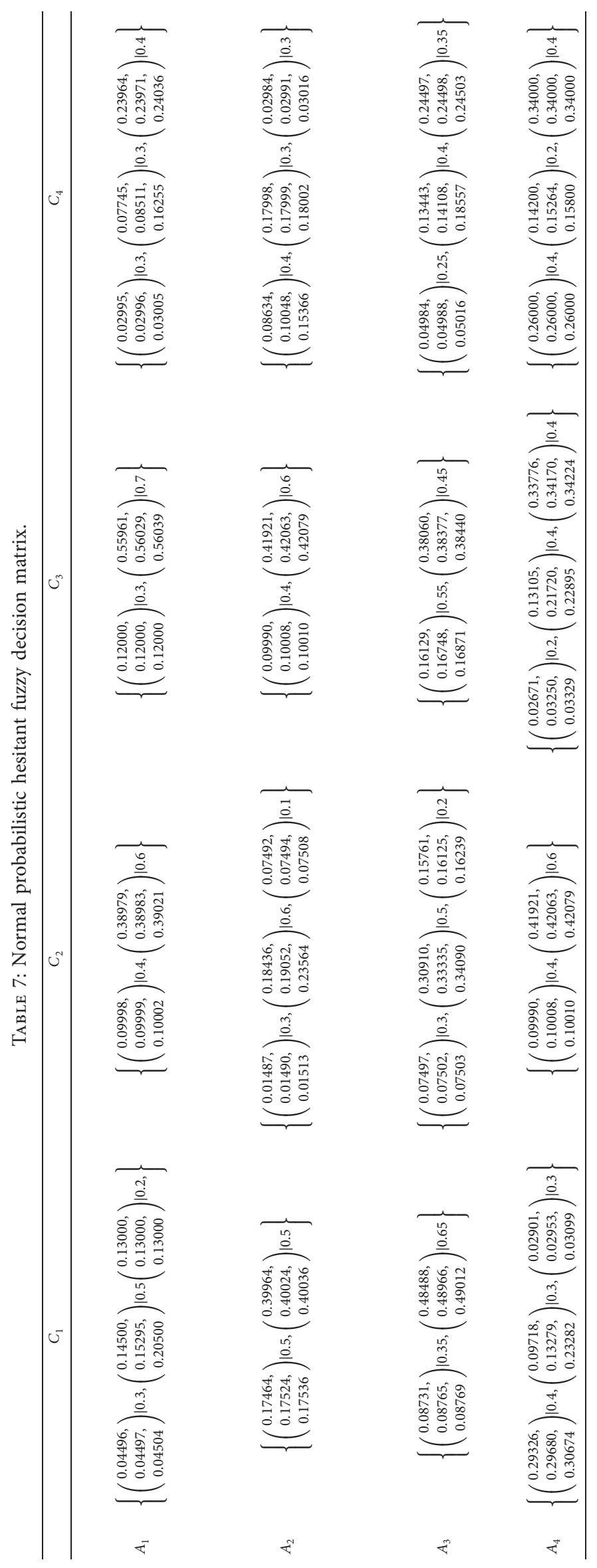


TABLE 8: The collective evaluation values of the alternatives and ranking.

\begin{tabular}{lccccc}
\hline & $A_{1}$ & $A_{2}$ & $A_{3}$ & $A_{4}$ & Ranking \\
\hline NWPHFWG & 0.1977 & 0.1741 & 0.2350 & 0.2398 & $A_{4}>A_{3}>A_{1}>A_{2}$ \\
NWPHFWA & 0.2438 & 0.2321 & 0.2701 & 0.2853 & $A_{4}>A_{3}>A_{1}>A_{2}$ \\
\hline
\end{tabular}

TABle 9: The decision results of the different methods.

\begin{tabular}{lccccc}
\hline & $A_{1}$ & $A_{2}$ & $A_{3}$ & $A_{4}$ & Ranking \\
\hline NWHFWG & 0.1185 & 0.0613 & 0.1622 & 0.1581 & $A_{3}>A_{4}>A_{1}>A_{2}$ \\
NWHFWA & 0.1904 & 0.2140 & 0.3046 & 0.4573 & $A_{4}>A_{3}>A_{2}>A_{1}$ \\
\hline
\end{tabular}

(3) Also, the operators given by [26] (NWHFWA and NWHFWG) are used in the above example, which provides different ranking on the same data, which is not practical. On the other side, the proposed operators (NWPHFWA and NWPHFWG) offer the same ranking, indicating that the proposed operators provide us more reliable and consistent results.

\section{Conclusions and Future Prospects}

In the decision-making process, the representation of uncertain information is proposed to enable the DMs to disclose their cognitive preferences fully. However, the limited knowledge of DMs leads to the fact that no complex information representation form can help DMs express all the preferred information about an alternative. Instead, it significantly increases the DMs psychological burden and time cost. Therefore, the purpose of this article is to obtain more accurate assessments from simple information. Therefore, to facilitate the DMs, we leave the dilemma of complex representation and try to find the hidden uncertain information from the original data provided by the DMs. To accomplish this objective, we propose a new representation tool, NWPHFS, to automatically find the hidden uncertain information of the original PHF information. The proposed NWPHFS is based on the assumption that human cognitive uncertainty can be considered a general fluctuation in a specific range that focuses on a value, the DM's uncertain feelings can appear objectively and realistically. In this paper, the essential theoretical knowledge of NWPHFS has been explained in detail:

(1) We propose some basic operational rules, score function, and distance measure between two NWPHFSs

(2) To aggregate the information, two aggregation operators are proposed, namely, normal wiggly probabilistic hesitant fuzzy weighted averaging and normal wiggly probabilistic hesitant fuzzy weighted geometric

(3) Based on NWPHFWA and NWPHFWG, a new MADM method is proposed to deal with MADM problems in a normal wiggly probabilistic context

(4) The effectiveness and feasibility of the proposed method are tested through an example of environmental quality assessment, and the comparative analysis revealed that the proposed method could offer more accurate and precise conclusions than the existing method

Future research can combine NWPHFS with some MADM methods considering preference relations, such as the TDM method and PROMETHEE method. Moreover, the Maclaurin Symmetric Mean and dual Maclaurin Symmetric Mean operators can be extended for the NWPHF environment. Simultaneously, we can further develop the MADM to the multiattribute group decision-making method and use this for different applications such as green supplier selection, robot selection, and environmental quality assessment.

\section{Data Availability}

No data were used to support this study.

\section{Conflicts of Interest}

The authors declare that they have no conflicts of interest.

\section{References}

[1] J. W. Gong, H. C. Liu, X. Y. You, and L. Yin, "An integrated multi-criteria decision making approach with linguistic hesitant fuzzy sets for E-learning website evaluation and selection," Applied Soft Computing, vol. 102, pp. 107-118, 2021.

[2] S. Faizi, T. Rashid, and S. Zafar, "A multi-criteria decisionmaking approach based on fuzzy AHP with intuitionistic 2tuple linguistic sets," Advances in Fuzzy Systems, vol. 2018, Article ID 5789192, 12 pages, 2018.

[3] L. A. Zadeh, "Fuzzy sets," Information and Control, vol. 8, no. 3, pp. 338-353, 1965.

[4] K. T. Atanassov, "Intuitionistic fuzzy sets," Fuzzy Sets and Systems, vol. 20, no. 1, pp. 87-96, 1986.

[5] J. M. Mendel and R. I. B. John, "Type-2 fuzzy sets made simple," IEEE Transactions on Fuzzy Systems, vol. 10, no. 2, pp. 117-127, 2002.

[6] K. T. Atanassov, "Interval valued intuitionistic fuzzy sets," in Intuitionistic Fuzzy Setspp. 139-177, Heidelberg, Germany, 1999.

[7] F. Xiao and W. Ding, "Divergence measure of pythagorean fuzzy sets and its application in medical diagnosis," Applied Soft Computing, vol. 79, pp. 254-267, 2019.

[8] V. Torra, "Hesitant fuzzy sets," International Journal of Intelligent Systems, vol. 25, no. 6, pp. 529-539, 2010. 
[9] B. Zhu, Decision Method for Research and Application Based on Preference Relation, Southeast University, Nanjing, China, 2014.

[10] S.-H. Xiong, Z.-S. Chen, and K.-S. Chin, "A novel MAGDM approach with proportional hesitant fuzzy sets," International Journal of Computational Intelligence Systems, vol. 11, no. 1, pp. 256-271, 2018.

[11] V. Torra and Y. Narukawa, "On hesitant fuzzy sets and decision," in Proceedings of the 18th IEEE International Conference on Fuzzy Systems, pp. 1378-1382, Jeju Island, South Korea, August 2009.

[12] Z. Zhang, "Hesitant fuzzy power aggregation operators and their application to multiple attribute group decision making," Information Sciences, vol. 234, pp. 150-181, 2013.

[13] D. Li, W. Zeng, and J. Li, "New distance and similarity measures on hesitant fuzzy sets and their applications in multiple criteria decision making," Engineering Applications of Artificial Intelligence, vol. 40, pp. 11-16, 2015.

[14] X. Liu, Z. Wang, S. Zhang, and H. Garg, "Novel correlation coefficient between hesitant fuzzy sets with application to medical diagnosis," Expert Systems with Applications, vol. 183, Article ID 115393, 2021.

[15] D. Li, W. Zeng, and Y. Zhao, "Note on distance measure of hesitant fuzzy sets," Information Sciences, vol. 321, pp. 103115, 2015.

[16] Z. Mu, S. Zeng, and T. Baležentis, "A novel aggregation principle for hesitant fuzzy elements," Knowledge-Based Systems, vol. 84, pp. 134-143, 2015.

[17] R. Wang and Y. Li, "Picture hesitant fuzzy set and its application to multiple criteria decision-making," Symmetry, vol. 10, no. 7, p. 295, 2018.

[18] S. Zhang, Z. Xu, and Y. He, "Operations and integrations of probabilistic hesitant fuzzy information in decision making," Information Fusion, vol. 38, pp. 1-11, 2017.

[19] J. Li, J. Wang, and J. Hu, "Multi-criteria decision-making method based on dominance degree and BWM with probabilistic hesitant fuzzy information," International Journal of Machine Learning and Cybernetics, vol. 10, pp. 1671-1685, 2019.

[20] Z.-X. Wang and J. Li, "Correlation coefficients of probabilistic hesitant fuzzy elements and their applications to evaluation of the alternatives," Symmetry, vol. 9, no. 11, p. 259, 2017.

[21] R. Krishankumar, K. S. Ravichandran, S. Kar, P. Gupta, and M. K. mehlawat, "Interval-valued probabilistic hesitant fuzzy set for multi-criteria group decision-making," Soft Computing, vol. 23, no. 21, pp. 10853-10879, 2019.

[22] R. Krishankumar, K. S. Ravichandran, A. H. Gandomi, and S. Kar, "Interval-valued probabilistic hesitant fuzzy set-based framework for group decision-making with unknown weight information," Neural Computing and Applications, vol. 33, pp. 2445-2457, 2020.

[23] Q. Noor, T. Rashid, and S. M. Husnine, "An extended TDM method under probabilistic interval-valued hesitant fuzzy environment for stock selection," PLoS One, vol. 16, no. 5, Article ID e0252115, 2021.

[24] Z.-S. Chen, C. Yu, K.-S. Chin, and L. Martínez, "An enhanced ordered weighted averaging operators generation algorithm with applications for multicriteria decision making," Applied Mathematical Modeling, vol. 71, pp. 467-490, 2019.

[25] S.-H. Xiong, Z.-S. Chen, J.-P. Chang, and K.-S. Chin, "On extended power average operators for decision-making: a case study in emergency response plan selection of civil aviation," Computers \& Industrial Engineering, vol. 130, pp. 258-271, 2019.
[26] Z. Ren, Z. Xu, and H. Wang, "Normal wiggly hesitant fuzzy sets and their application to environmental quality evaluation," Knowledge-Based Systems, vol. 159, pp. 286-297, 2018.

[27] Z. Liu, L. Li, X. Wang, and P. Liu, "A novel multiple-attribute decision making method based on power muirhead mean operator under normal wiggly hesitant fuzzy environment," Journal of Intelligent \& Fuzzy Systems, vol. 37, no. 5, pp. 7003-7023, 2019.

[28] P. Liu, H. Xu, and W. Pedrycz, "A normal wiggly hesitant fuzzy linguistic projection-based multiattributive border approximation area comparison method," International Journal of Intelligent Systems, vol. 35, no. 3, pp. 432-469, 2020.

[29] C. Yang, Q. Wang, W. Peng, J. Zhang, and J. Zhu, "A normal wiggly pythagorean hesitant fuzzy bidirectional projection method and its application in EV power battery recycling mode selection," IEEE Access, vol. 8, pp. 62164-62180, 2020.

[30] S. Narayanamoorthy, L. Ramya, D. Baleanu, J. V. Kureethara, and V. Annapoorani, "Application of normal wiggly dual hesitant fuzzy sets to site selection for hydrogen underground storage," International Journal of Hydrogen Energy, vol. 44, no. 54, pp. 28874-28892, 2019.

[31] P. Liu and P. Zhang, "Normal wiggly hesitant fuzzy TODIM approach for multiple attribute decision making," Journal of Intelligent \& Fuzzy Systems, vol. 39, no. 1, pp. 627-644, 2020.

[32] P. Liu and P. Zhang, "A normal wiggly hesitant fuzzy MABAC method based on CCSD and prospect theory for multiple attribute decision making," International Journal of Intelligent Systems, vol. 36, no. 1, pp. 447-477, 2021.

[33] M. Xia and Z. Xu, "Hesitant fuzzy information aggregation in decision making," International Journal of Approximate Reasoning, vol. 52, no. 3, pp. 395-407, 2011.

[34] R. R. Yager, "On ordered weighted averaging aggregation operators in multicriteria decision making," IEEE Transactions on Systems, Man, and Cybernetics, vol. 18, no. 1, pp. 183-190, 1988.

[35] W. Guiwu, W. Hongjun, Z. Xiaofei, and L. Rui, "Hesitant triangular fuzzy information aggregation in multiple attribute decision making," Journal of Intelligent \& Fuzzy Systems, vol. 26, no. 3, pp. 1201-1209, 2014.

[36] X. Zhao, R. Lin, and G. Wei, "Hesitant triangular fuzzy information aggregation based on Einstein operations and their application to multiple attribute decision making," Expert Systems with Applications, vol. 41, no. 4, pp. 1086-1094, 2014. 